\title{
Study on Doppler Correction in Demodulation of Digital
}

\section{Signals}

\author{
Xu Xingyuan, Xia Lili, Liu Sifang \\ China Satellite Maritime Tracking and Control Department, Jiangyin, 214431, China
}

Abstract: When the spacecraft is moving at high speed, the signal obtained by the control station always exists Doppler shift, which is reflected in the DQPSK signal. In order to make the digital signal demodulate the correct information, this paper analyzes the influence of Doppler on demodulation, and studies the modified Doppler method based on DSTFT to ensure that Doppler interference does not interfere with the normal demodulation.

Key words: Doppler; PCM-DQPSK; Demodulation

\section{Introduction}

DQPSK signal demodulation, coherent demodulation to restore the same frequency in-phase carrier as the goal, the research focused on the carrier recovery algorithm, including carrier closed-loop recovery algorithm and open-loop recovery algorithm. Closed-loop recovery algorithm often uses digital phase-locked loop to achieve the carrier capture and tracking, such as loose tail carrier synchronization technology, based on DDS to achieve digital phase-locked loop carrier synchronization, Costas ring. In the area of non-coherent demodulation, the focus of attention in recent years has focused on high-performance differential demodulation. But no matter what demodulation method, have to go through Doppler correction, so as to demodulate the correct data.

\section{Single-frequency DFT operation}

FFT algorithm can be used to improve the computing efficiency, but the speed of operation is limited. For a time-domain signal $\mathrm{x}(\mathrm{n})$, a single point-N FFT computes the $\mathrm{X}(\mathrm{k})$ value for all $\mathrm{N}$ points in the frequency domain. If only the $\mathrm{X}(\mathrm{k})$ value of a discrete point needs to be known, , Then there is no need to calculate the FFT by the full spectrum, and can be directly calculated using the DFT frequency points of the discrete frequency value, called the single-frequency DFT operation. In DSTFT-based demodulation, we are interested only in the DFT phase of the center frequency in the whole frequency domain. Therefore, single-frequency DFT operations can be used. In this way, the amount of calculation in each window becomes one of the original $\mathrm{N}$, greatly simplifying the operation.

Traditional FFT analysis of the frequency accuracy is considered only $1 / T_{s}$, but in fact, the window after the discrete-time signal Fourier transforms:

$$
X\left(e^{j \omega}\right)=\sum_{n=0}^{N-1} w(n) x(n) e^{-j n \omega}
$$

The Fourier transform of the signal $\mathrm{x}(\mathrm{n})$ is a continuous function of $\mathrm{w}$, and as long as a given $\mathrm{w}$ is given, $\mathrm{X}(\mathrm{w})$ can be calculated. (1) is transformed into a discrete Fourier transform according to the nature of the circular convolution:

$$
X_{w}(k)=\sum_{n=0}^{N-1} w(n) x(n) e^{-j \frac{2 \pi k n}{N}}, \quad k=0,1,2 \cdots N-1
$$

It can be seen that Eq. (2) is exactly the result of equal interval discretization of equation (1), and k can take any consecutive value from 0 to $(\mathrm{N}-1)$, not just integer values. In this way, we can obtain the continuous value to obtain the corresponding DFT value of any frequency value, in order to eliminate the fence effect.

Therefore, for DSTFT demodulation, if the carrier frequency is known, the Fourier transform of the frequency point $k_{c}=N f_{c} / f_{s}$ can be directly calculated and the phase of the DFT value corresponding to this frequency point can be obtained. This can be a small amount of computation, but also can get a higher frequency resolution.

In addition, the direct-signal single-frequency Fourier transform also has the advantage that the number of signal samples for DFT operation can be any value, without having to be an integer power of 2 as in the FFT. Therefore, 
when the length $\mathrm{M}$ of the DSTFT calculation data window is determined, the number of arithmetic points $\mathrm{N}$ of the Fourier transform is equal to $\mathrm{M}$, and it is not necessary to perform the zero padding process.

\section{Effect of Doppler Shift on Demodulation}

Assuming that there is a Doppler frequency offset, the $\pi$ / 4-DQPSK signal is expressed as:

$$
S(n)=A \cos \left[2 \pi\left(f_{c}+f_{d}\right) n / f_{s}+\Delta \theta_{\mathrm{k}}\right]
$$

According to the demodulation principle of DSTFT, a fixed-length sliding window is designed. From the first sampling point of the signal sequence, the data is truncated to do single-frequency DFT operation, and then the differential phase is calculated. In this process involves the central frequency of the two links, one is the single-frequency DFT operation, and the other is the differential phase calculation, which is the Doppler shift will have an impact on the algorithm lies. The errors caused by the DSTFT demodulation when there is a Doppler shift are deduced from the formula.

The single-frequency DFT calculates only the Fourier transform of $k_{c}=N f_{c} / f_{s}$, and the center frequency of the carrier at this time should be adjusted to $\mathrm{f}=\mathrm{fc}+\mathrm{fd}$ due to Doppler. By the phase resolution formula available, when there is Doppler frequency shift, if still with the original carrier frequency to calculate the single-frequency DFT will cause the phase error is:

$$
\Delta \varphi_{1}=\frac{\pi}{N} \Delta k_{d}(M-1)=\frac{\pi}{N} \cdot \frac{f_{d} N}{f_{s}} \cdot(M-1)=\frac{\pi(M-1) f_{d}}{f_{s}}
$$

The phase difference between symbols is:

$$
\Delta \theta_{k}=\Delta \varphi_{k}-2 \pi\left(f_{c}+f_{d}\right) l / f_{s}
$$

And if we still use a fixed carrier frequency to calculate the differential phase sequence, it will cause the error:

$$
\Delta \varphi_{2}=\frac{2 \pi f_{d} l}{f_{s}}=\frac{2 \pi f_{d} \cdot 2 f_{s} / R_{b}}{f_{s}}=\frac{4 \pi f_{d}}{R_{b}}
$$

Therefore, the maximum differential phase error due to Doppler is:

$$
\Delta \varphi=\Delta \varphi_{1}+\Delta \varphi_{2}=\frac{\pi(M-1) f_{d}}{f_{s}}+\frac{4 \pi f_{d}}{R_{b}}=f_{d}\left[\frac{\pi(M-1)}{f_{s}}+\frac{4 \pi}{R_{b}}\right]
$$

The difference phase error may lead to miscarriage of justice. Since the minimum phase error caused by misjudgment is $\pi / 4$, it is assumed that $\Delta \varphi=\frac{\pi}{4}$ and the relevant parameter is substituted into equation (7) for calculation. The Doppler at the time of correct demodulation under the actual data index can be obtained. In other words, theoretically, the DSTFT demodulation is performed using a generalized cosine window of length 8 (one carrier period) for the signal (no noise) of the actual data in the task if there is a Doppler shift, it will not produce errors, the conclusion has been confirmed by computer simulation. Conversely, if the Doppler shift is outside this range, appropriate corrections are required.

\section{Correction of Doppler Shift}

There are many ways to correct the Doppler shift, and for the demodulation of the DQPSK signal, the Doppler shift is mainly reflected in the estimation of the carrier frequency, which can be converted into a single carrier frequency signal of the frequency measurement. Frequency measurement methods are many, high-precision frequency measurement results is endless. As the DSTFT demodulation algorithm itself allows a certain range of Doppler frequency shift exists, and taking into account the problem of system efficiency, a signal frequency estimation algorithm is designed. When the range of Doppler frequency shift is known, or the frequency shift measurement accuracy is also required, the center frequency of the signal can be estimated by DSTFT operation.

Assuming $\mathrm{x}(\mathrm{n})$ is a signal with a single frequency, DSTFT transforms the signal $\mathrm{x}(\mathrm{n})$ according to the relevant 
definition, the search range of the set frequency and the search frequency step:

$$
S_{x}\left(n, f_{i}\right)=\sum_{m=0}^{M-1} x(m) W(n-m) e^{-j 2 \pi \cdot \frac{f_{i} \cdot M m}{f_{s}}}
$$

In formula, $f_{i}=f-f_{d}+(i-1) \Delta f, i=1 \sim\left(2 f_{d} / \Delta f+1\right), \mathrm{M}$ is the window length when the frequency is searched.

According to formula (8), for each frequency, a DFT amplitude sequence can be obtained, the average value of the sequence as the frequency of the DFT amplitude, then the maximum value of the corresponding frequency is the signal $\mathrm{x}(\mathrm{n})$ frequency.

According to different SNR conditions, Figure 1 shows the normalized mean square error using rectangular window. The signal parameters are carrier frequency $2.1 \mathrm{MHz}$, sampling rate $16 \mathrm{Msps}$, code rate $1.536 \mathrm{Mbps}$, window length $\mathrm{M}$ is 10,15 and 20 sampling points.

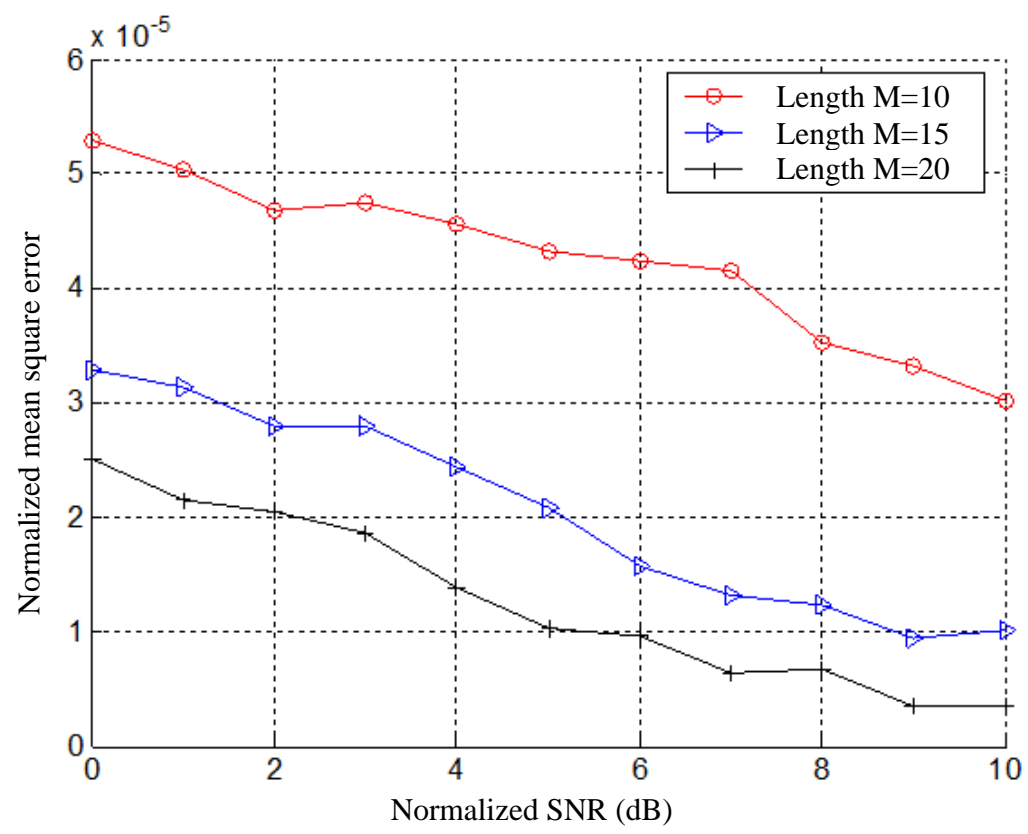

Figure 1. DSTFT frequency measurement error

It can be seen from Figure 1 that the normalized mean square error of the signal frequency using DSTFT estimation is small, and the frequency error is related to the window length. The longer the DSTFT window, the higher the frequency measurement accuracy.

\section{Summary}

In this paper, we mainly study the effect of Doppler on demodulation, and study the Doppler synchronization method based on DSTFT to ensure that Doppler interference is not disturbed to the normal demodulation of the signal.

\section{Reference:}

[1] L.Erup, F,M,Gardner, interpolation in digital modems-part II. Implementation and performance, IEEE. Trans. Com, Jun.2013, 41(6):998-1008.

[2] Chuang J.C, Sollenberger N.R, Burst coherent demodulation with combined symbol timing frequency offset estimation, and diversity selection. IEEE,Trans.Com, 2011, 39(7):1157-1164.

[3] Sollemberger N.R, Chuang J.C, Low Overhead symbol timing and carrier recovery for TDMA portable radia systems. IEE, Trans.Com, 2010, 38(10):1886-1892.

[4] G.Ascheid, M.oerder, H.meyr, An All Digital Receiver Architecture for Bandwidth Efficient Transmussion at 
high Rates, IEEE, Trans. Com, Aug.2009, 37(8):804-813.

[5] D.Divsalar and M. K.Simon. Multiple-symbol differential detection of MPSK[J]. IEEE Trans, Communm, 2010, 38(3):300-308.

[6] H.Leib, S.Pasupathy. The phase of a vector perturbed by Gaussian noise and differentially coherent receivers[J]. IEEE Trans. Inform, Theory, 2008, 34(6):1491-1501.

[7] Hamamoto N. Differential detection with IIR filter for improving DPSK detection performance[J]. IEEE Transaction on Communications, 2006:959-966.

[8] Wong D P C, Mathiopoulos P T. Nonredundant error correction analysis and evaluation of differentially detected $\pi / 4$-DQPSK systems in a combined CCI and AWGN environment[J]. IEEE Transactionon Vehicular Technology, 2011(1):35-471

[9] Spilker. J.J. Digital Communications by Satellite[C], Prentice-Hall, Inc. Englewood Cliffs, N.J, 2007.

[10] Jeong Eun, Lee, Seung Hee Choi, Beomsup Kim. Basedband clock recovery algorithm for $\pi / 4$-qpsk modulated signals[C]. IEEE Vehicular Technology Conference, 2007:1729-1733.

[11] Beomsup Kim,Jun Mo Koo, Joonsuk Lee, etc. Non-Data-Aided Timing Recovery Algorithm for $\pi / 4-Q P S K$ Modulated signals[C]. IEEE International Conference on Communications, 2010, 1(3):392-396.

[12] Dongmin Lim. A Modified Gardner Detector for Symbol Timing Recovery of M-PSK Siganls[J]. IEEE Transaction on Communications, 2013, 52(10). 